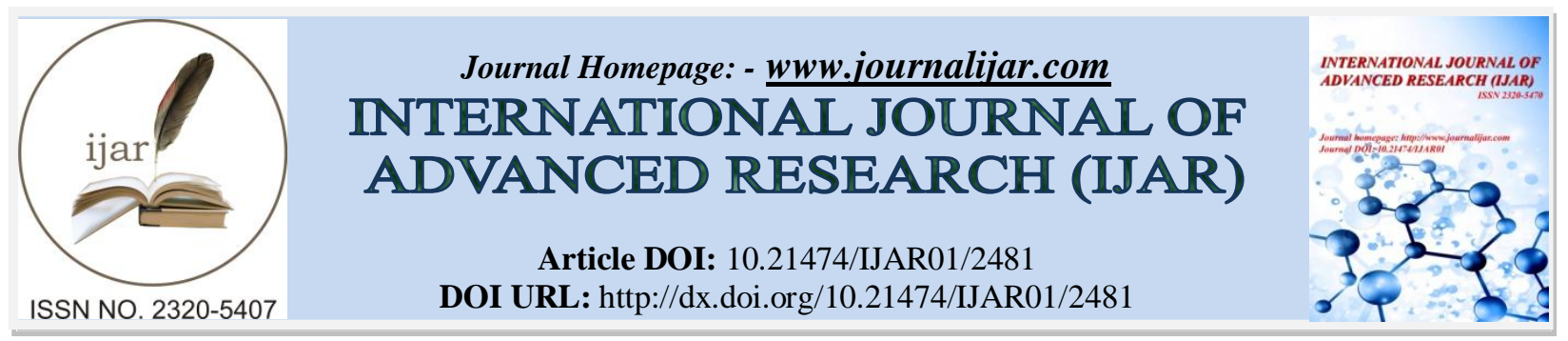

RESEARCH ARTICLE

\title{
CHALLENGES OF WSN FACED IN MOBILITY SCENARIOS.
}

Sai madhavi $D^{1}$ and Dr Y. S. Kumar Swamy ${ }^{2}$.

1. Research Scholar, Rayalaseema University, Kurnool and Associate Professor CSE Dept, RYMEC, Ballari.

2. Guide, Rayalaseema University, Kurnool and Prof and Dean (R\&D) CSE Dept, NCET , Bengaluru.

\section{Manuscript Info}

Manuscript History

Received: 23 October 2016

Final Accepted: 21 November 2016

Published: December 2016

Key words:-

TCP/IP, MAC, Energy

\section{Abstract}

Among these necessities, continuous correspondence is one of the huge research challenges in remote sensor systems on the grounds that the majority of inquiry messages convey time data. To meet this necessity, as of late a few constant medium get to control conventions have been proposed for remote sensor organizes in the writing since holding up time to share medium on every hub is one of fundamental hotspot for end-to-end delay. There exists a considerable measure of medium get to control (MAC) conventions for remote sensor systems. Be that as it may, they consider essentially vitality proficiency as opposed to idleness which is critical in postpone delicate portable sensor systems. These sensor hubs should work with their constrained and none renew capable vitality assets. Despite the fact that vitality preservation in correspondence can be performed in various layers of the TCP/IP convention suit, vitality protection at MAC layer is observed to be the best one because of its capacity to control the radio straightforwardly. In this manner, to guarantee a seemingly perpetual system of remote conveying sensors, we need a MAC convention that can enhance vitality effectiveness by boosting rest term, minimizing inactive listening and catching, and taking out crash of parcels. This paper introduces an audit of the latest works with respect to MAC conventions for uses of remote sensor systems. In the first place we display talks on radio vitality show, vitality utilization measuring and the sensor organize properties that are essential for the plan of MAC layer conventions. Second we portray the few accessible vitality effective MAC conventions for sensor systems underscoring their vitality sparing techniques.

Copy Right, IJAR, 2016,. All rights reserved.

\section{Introduction:-}

Wellsprings of vitality wastefulness in WSNs came as a consequence of various operations of sensor hubs. Occasions must be identified along these lines detecting is one of the primary wellsprings of vitality exhaustion. Another is steering which decides how the detected information is handed-off back to the sink. Preparing of detected information is additionally a vitality depleting process in WSNs. Transmitting every single crude data expends significant vitality and in addition transmission data transfer capacity and consequently not perfect. Guaranteeing minimal effort end-to-end correspondence with satisfactory information conveyance deferral is the real test confronted by WSNs. These issues are intensified in LWSNs, on the grounds that the straight topology restricts the 
quantity of neighbors and in this way the conceivable transmission courses, which makes information misfortune more probable than in traditional WSNs. For the most part, LWSNs experience the ill effects of uneven information movement among hubs, for example hubs nearer to the sink as a rule are more congested than the hubs found further away. This is expected to the multi jump correspondence approach utilized for transmitting information from hubs that cannot be achieve the sink specifically. Subsequently, information transmission disappointments increment fundamentally because of blockage alongside an expansion in correspondence delays because of successive retransmissions.

\section{Survey of WSN MAC Protocols:-}

[1]. Remote sensor systems (WSNs) have turned into a dynamic research region for the scientists because of their generally utilized scope of application potential in regions, for example, target recognition and following, natural checking, mechanical process observing, and strategic frameworks. As a rule, after the sending of sensor hubs in the unsafe, threatening or remote regions, these sensor hubs chip away at their own. These sensor hubs should work with their restricted and none recharge capable vitality assets. Despite the fact that vitality preservation in correspondence can be performed in various layers of the TCP/IP convention suit, vitality protection at MAC layer is observed to be the best one because of its capacity to control the radio straightforwardly. Hence, to guarantee a seemingly perpetual system of remote imparting sensors, we need a MAC convention that can enhance vitality proficiency by expanding rest length, minimizing inert listening and catching, and disposing of impact of bundles. This paper displays a survey of the latest works concerning MAC conventions for utilizations of remote sensor systems. To start with we exhibit exchanges on radio vitality demonstrate, vitality utilization measuring and the sensor arrange properties that are vital for the plan of MAC layer conventions. Second we depict the few accessible vitality proficient MAC conventions for sensor systems accentuating their vitality sparing techniques. These energy efficient Macintosh conventions are basically separated into two gatherings, i.e. synchronous and non concurrent MAC conventions. At long last, we talk about some future research issues in the plan of MAC convention for WSNs.

[2]. There exists a considerable measure of medium get to control (MAC) conventions for remote sensor systems. Be that as it may, they consider predominantly vitality proficiency as opposed to inertness which is critical in defer touchy versatile sensor systems. Accordingly, another MAC convention called MS-MAC is proposed reasonable to both stationary and versatile sensor systems. MS-MAC utilizes a versatile edge structure enhanced for understood convention S-MAC to beat the execution issues brought on by the parcels idleness in the portable environment. The determination of screen hubs and occasional booking of synchronization bundles are utilized to anticipate the speed of versatile hubs, which can spare the vitality utilization and minimize latencies. Our studies demonstrate that under static situations, the MS-MAC conduct comparable with MS-MAC. Be that as it may, MS-MAC can expand the system throughput and diminish the inactivity fundamentally without more vitality misfortune in examination with S-MAC in versatile environment.

[3]. As remote sensor organize gets to be distinctly inescapable, new necessities have been persistently developed. However, a large portion of research endeavors in remote sensor system are centered around vitality issue since the hubs are generally battery-fueled. Among these necessities, ongoing correspondence is one of the enormous research challenges in remote sensor systems on the grounds that the majority of question messages convey time data. To meet this prerequisite, as of late a few continuous medium get to control conventions have been proposed for remote sensor arranges in the writing since holding up time to share medium on every hub is one of primary hotspot for end-to-end delay. In this paper, we first present the particular prerequisite of remote sensor ongoing MAC convention. At that point, a gathering of late remote sensor continuous MAC conventions are studied, ordered, and depicted underlining their preferences and hindrances at whatever point conceivable. At last we exhibit a discussion about the difficulties of current remote sensor ongoing MAC conventions in the writing, and demonstrate the conclusion at last.

[4].A Mobile Sensor Network is a geologically conveyed arrange which owes its name to the nearness of versatile sink or sensor hubs inside the system. In this paper we will exhibit and talk about the vitality productive dispute based and booked based medium get to control (MAC) conventions for versatile sensor systems (MSNs). The vast majority of the MAC conventions, for example, T-MAC, D-MAC, and the more generally used SMAC proposed for remote sensor systems accept sensors to be stationary after arrangement, which as a rule give terrible system execution in situations including versatile sensors. Taking care of portability in remote sensor arranges in a vitality productive way is another test. We exhibit new MAC layer ways to deal with bolster portability in WSNs, for 
example, MS-MAC stretches out S-MAC to incorporate versatility mindfulness by diminishing this rest span when versatility is identified. S-MAC with Extended Kalman channel (EKF) decreases versatility brought about misfortunes by foreseeing the ideal information outline measure for every transmission. M-LMAC (Mobile LMAC) as a TDMA-based MAC convention which can bolster ceaseless portability in the remote sensor systems. MMAC uses a dynamic versatility versatile edge time to improve TRAMA, a booked based convention, with portability expectation. In like manner, G-MAC uses TDMA for group based WSNs by consolidating the benefits of dispute and conflict free MACs. The greater part of the above MAC conventions are checked on in detail.

[5]. WSN is generally utilized as a part of checking, following and military applications. It has restricted assets like battery limit, data transmission and handling unit. In WSN comprises of is a hubs and the base station for system arrangement and hub having the preparing unit, correspondence unit and the power unit. For the most part the hub transmits information to sink which has abundant assets. Also WSN devour more vitality for transmission as opposed to handling and that inspire specialists to work for the tradeoff between lifetime of the system, throughput and dormancy. Information correspondence is connected with the system and MAC layer. Macintosh is in charge of radio getting to, hub wake up and rest timetable, Listening and detecting assignment. Likewise with perfect detecting and listening devour more vitality than information correspondence and disgraceful wake-up and rest plan diminish throughput and increment idleness. The point of this paper is to break down different MAC convention and furnish an outline idea with QoS parameters.

\section{Methodology:-}

Customarily, the vitality utilization is considered as the most essential research issue in WSNs. S-MAC and T-MAC, are the delegate conventions used to diminish the vitality utilization in WSNs. They utilize the operation of lowobligation cycle to protect the vitality of the sensor hubs. By utilizing the operation of low-Duty-cycle, sensor hubs can rest when the hubs require not speak with each other and decrease vitality utilization. The conventions likewise propose an idea of virtual bunch to lessen control overhead and permit activity versatile wake-up. Despite the fact that these conventions address the fundamental issues of sparing vitality, these conventions may prompt to a long parcel delay because of a rest period, if the conventions are utilized as a part of MWSNs.

The most generally utilized MAC convention for sensor systems is S-MAC. S-MAC presented a low-obligation cycle operation in multi-bounce remote sensor systems, where the hubs invest the greater part of their time in rest mode to lessen vitality utilization. Papers on T-MAC demonstrated that S-MAC does not perform well with variable movement loads. T-MAC presented movement versatile element rest and wakeful periods for sensor hubs. These conventions expect static systems with the emphasis on vitality protection. The casing time in S-MAC and T-MAC is settled. We acquaint super edge all together with synchronize portable hubs.

\section{Structure of MAC:-}

Sensor hubs embrace a similar rest wake up timetable to spare vitality when the hubs keep up correspondences between hubs in WSNs. Figure 1 demonstrates a case of the S-MAC structure comprising of tune in, rest, and Neighbor Discovery Protocol (NDP). Planning is a standout amongst the most confused errands in WSNs. S-MAC is a dispute based MAC convention, exceptionally intended for WSNs. Its primary thought is that the hubs rest and listen intermittently. Outline decides an ideal opportunity to rest and tune in. Casing is a consistent day and age. Since neighboring hubs, which can convey inside one bounce, can impart inside the same listen period, they should take a similar timetable. As it were, neighboring hubs ought to be synchronized to rest and wake up intermittently. The listen time of every casing relegates a particular time for a synchronization parcel, called SYNC bundle. So as to look after synchronization, every hub communicates occasionally a SYNC bundle that contains its own timetable, for each 10 outlines in this illustration. In the event that the neighboring hubs get SYNC messages, they will upgrade their timetable tables, which is a period table for the planning interchanges. At the point when a SYNC bundle is meddled or debased, or a SYNC parcel is deferred due to the occupied of systems, two hubs regularly neglect to perceive each other. For this situation, the Neighbor Discovery Protocol (NDP) is designated (for 10-second synchronization period at regular intervals. All hubs must keep the listen state amid this NDP without tumbling to the rest state.

In WSNs, a bunch is oftentimes utilized for a proficient or viable information handling. By and large, a bunch is fabricated in light of the land area. As it were, a group comprises of adjacent sensor hubs. The previously mentioned synchronization technique does not require a sound calendar for the whole system. In any case, synchronization ought to be done inside a clustere. For interchanges between neighboring groups, the hubs on the bunch fringes must 
know about the listen time of the neighboring groups, and additionally the listen time of their own bunches. S-MAC synchronization calculation functions admirably under static systems where the era and break of associations don't happen every now and again. Under a situation where portable sensor hubs exist, nonetheless, it can bring about as a long slack as the synchronization time frame that desires 8 seconds at regular intervals when a versatile hub enters another group, gets a SYNC parcel, and synchronize with the new timetable. Along these lines, a hub can be detached from the system up to 110 seconds sitting tight for synchronization. Such a parcel postponement can prompt to a poorer execution, which can be considerably more genuine for time basic applications.

\section{Simulation Analysis:-}

In this area, System can assess the execution of reproduction. System are utilizing the $\mathrm{x}$ _graph for assess the execution. System utilize some assessment measurements, Packet conveyance proportion: it is the proportion of the quantity of parcel got at destination and number of bundle sent by the source. Throughput: The throughput metric spoke to the framework information efficiency amid the system operation. Framework throughput was spoken to by the measure of information that was conveyed from a source to a destination amid a timeframe. End-to-End delay: the normal time taken for a parcel to be transmitted from source to destination, Energy level :- number of vitality devoured when the information ought to be transmitted. Vitality Consumption: The vitality utilization per round is the total of vitality devoured per round. System in this manner consider the vitality utilization as the vitality dispersed in transmitting and accepting parcels

\section{Conclusion:-}

We have talked about the qualities and working rule of various MAC conventions. Planning a MAC convention which can enhance vitality effectiveness to develop arrange lifetime in remote sensor systems is a testing issue. It is fundamentally due to stringent asset requirement both in sensor hubs and in remote media. As of late there are different vitality proficient MAC conventions both synchronous and non concurrent conventions for the WSNs that have been proposed by the specialists are exhibited in this paper. The plan of a streamlined MAC convention for vitality productivity likewise relied on upon the real application. Be that as it may, there is no particular MAC convention for the WSNs have been acknowledged as a standard. One reason for this is the MAC convention in general will be application subordinate. Another reason is the absence of institutionalization at physical layer and the sensor equipment. Accordingly, it will be hard to have one standard MAC convention which will work for all conceivable WSN applications. In this way, still a great deal of work needs to done in working out a MAC convention which will adjust its conduct in view of the applications.

\section{References:-}

1. Wireless Sensor Network: MAC Survey Patel Rinkuben N PhD scholar, School of Computer Science, RK University Teaching Assistant, Department of Computer Science, VNSGU, Surat, Gujarat, India

2. Dr. Nirav V. Bhatt Assistant Professor, MCA Department, RK University, Rajkot

3. Gujarat, India

4. Energy Efficient MAC protocols for Mobile SensorNetworksIsha Batra Ritu Malik Meenakshi MittalMaster's in Engineering, Master's in Engineering Master's in EngineeringPEC, sec-12 PEC, sec-12 PEC, sec-12Chandigarh Chandigarh Chandigarh

5. A Survey on Real-Time MAC Protocols in Wireless Sensor Networks Zheng Teng, Ki-Il Kim* Department of Informatics, Research Institute of Computer and Information Communication, Gyeongsang National University, Jinju, Korea E-mail: kikim@gnu.ac.kr Received January 13, 2010; revised February 19, 2010; accepted March 10, 2010.

6. A Mobility Aware and Low Latency Mac Protocol for Mobile Wireless Sensor Network with the Improvement of S_MAC Bin Zeng1 and Rui Wang2 1Department of Management Engineering, University of naval Engineering, China 2University Library, Department of Training, University of Naval Engineering, China

7. A Review on Medium Access Control Protocols forWireless Sensor NetworksRakesh Bisht 1, Abhishek Kumar Singh 2 Jai Prakash Bharti 3, Gourav Kumar 41, 2 Assist. Prof., Department of Computer Science and Engineering, ANACEMS, Bareilly3,4 Student,Computer Science and Engineering, ANACEMS, Bareilly1rakeshbisht534@gmail.com,2abhisheks.532@gmail.com,3engg.jai.cs@gmail.com, gouravrathore23@gmail.com

8. Sai Madhavi D, Kumarswamy, Y. S."Mechanisms Supporting Mobility in WSNs."

9. Advances in Computer Science and InformationTechnology. Networks and Communications.

10. Springer Berlin Heidelberg, 2012. 368-371.

11. Sai Madhavi D Swamy, YS Kumar. "OptimalLoad and Mobility Aware MAC Protocol for

12. Wireless Sensor Networks." International Journalof Computer Applications 63.22 (2013). 